\title{
Geschichte der Fiktionalität
}

Diachrone Perspektiven auf ein kulturelles Konzept

\author{
Herausgegeben von \\ Johannes Franzen, \\ Patrick Galke-Janzen, \\ Frauke Janzen, \\ Marc Wurich
}




\section{Umschlagabbildung:}

Johann Heinrich Zedler:

Großes Universal-Lexicon aller Wissenschaften und Künste, $1731-1754$

Bibliografische Information der Deutschen Nationalbibliothek Die Deutsche Nationalbibliothek verzeichnet diese Publikation in der Deutschen Nationalbibliografie; detaillierte bibliografische Daten sind im Internet über http://dnb.d-nb.de abrufbar.

(C) Ergon - ein Verlag in der Nomos Verlagsgesellschaft, Baden-Baden 2018

Das Werk einschließlich aller seiner Teile ist urheberrechtlich geschützt.

Jede Verwertung außerhalb des Urheberrechtsgesetzes bedarf der Zustimmung des Verlages.

Das gilt insbesondere für Vervielfältigungen jeder Art, Übersetzungen, Mikroverfilmungen und für Einspeicherungen in elektronische Systeme.

Gedruckt auf alterungsbeständigem Papier.

Satz: Matthias Wies

Umschlaggestaltung: Jan von Hugo

www.ergon-verlag.de

ISSN 2365-8851

ISBN 978-3-95650-????? (Print)

ISBN 978-3-95650-???-? (ePDF) 


\section{Inhaltsverzeichnis}

Johannes Franzen/Patrick Galke-Janzen/Frauke Janzen/Marc Wurich

Geschichte der Fiktionalität. Zur Einleitung

Benjamin Gittel

Fiktion und Genre. Systematische Überlegungen zu ihrem Verhältnis als Beitrag zur Historisierung und ,Kulturalisierung' von Fiktionalität

Wolfgang Rösler

Die Erfahrung dramatischer Dichtung und die Ausarbeitung

eines Konzepts von Fiktionalität im antiken Griechenland

Henrike Manuwald

Der Drache als Herausforderung für Fiktionalitätstheorien.

Mediävistische Überlegungen zur Historisierung von ,Faktualität

Sebastian Kleinschmidt/Verena Spohn

Was ein Rahmen möglich macht. Die mittelalterliche Vision als

,Spielraum` des Fiktionalen

Isabel Toral-Niehoff

Erzählen im arabischen adab. Zwischen Fiktionalität und Faktualität

Roman Kubn

„A just (Hi-)Story of Fact“. Peritextuelle Fiktionsmarkierungen

im Zeitalter des „Rise of the Novel“

Christopher Meid

Roman und Historie. Zur Wertung von Fiktionalität in der

Romantheorie der Aufklärung.

Frank Zipfel

Madame de Staëls Essai sur les fictions vor dem Hintergrund damaliger und heutiger Fiktionstheorien.

Mathis Lessau

Wilhelm Dilthey und das Paradoxon der Fiktion 
Marc Wurich

Mögliche Halbwelten. Heteroreferentialität und Diskurshybridisierung in naturalistischen Milieudarstellungen.....

Françoise Lavocat

Kontrafaktische Narrative in Geschichte und Fiktion.

Johannes Franzen

Fiktionskritik. Überlegungen zur ,Unwahrheit

des literarischen Erfindens

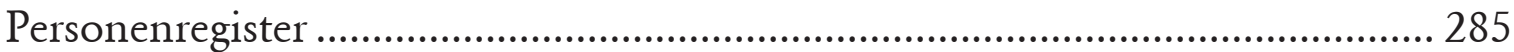




\section{Geschichte der Fiktionalität.}

\section{Zur Einleitung}

\section{Johannes Franzen, Patrick Galke-Janzen, Frauke Janzen, Marc Wurich}

In seinem einflussreichen Aufsatz „Zur historischen Genese der Scheidung von Fiktion und Realität" diagnostiziert Hans Robert Jauss die fundamentale Historizität des Fiktionalitätskonzepts:

Sowohl in der antiken wie dann auch wieder bei der Neubildung der mittelalterlichen Literatur ist die uns so selbstverständliche Scheidung von Fiktion und Realität nicht von Anbeginn gegeben, sondern erst vergleichsweise spät bezeugt. Die Nichtunterscheidung von Fiktion und Realität ist vielmehr auf den älteren Literaturstufen geradezu ein Aspekt ihrer für uns befremdlichen Alterität. Zeitpunkt, Anlaß, gesellschaftliche und psychologische Bedingungen der Genese des Fiktionsbegriffs, der unser Verständnis von fiktionaler Dichtung trägt, sind mit Worten noch wenig erforscht. ${ }^{1}$

Jauss entwirft hier die Vorstellung einer evolutionären Geschichte der Fiktion, verstanden als die Technik der literarischen Erfindung: Demnach muss die Literaturgeschichte das Konzept ,Fiktionalität ${ }^{\varsigma}$ und damit verbundene Produktionsund Rezeptionspraktiken erst hervorbringen, als Telos eines Fortschrittsnarrativs, das dann im autonomistischen Fiktionsverständnis der Moderne seine Erfüllung findet. So verstanden, zeigt sich die Geschichtlichkeit der Fiktionalität also vor allem darin, dass ,ältere Literaturstufen' die Trennung von Fiktion und Realität ,noch nicht ${ }^{\varsigma}$ entwickelt haben, dazu aber potentiell in der Lage sind - Fiktionalität erscheint als etwas, das in einem historischen Prozess erst ,erfunden' werden muss. Diese Entwicklungsgeschichte ist in der bisherigen Forschung recht weit verbreitet, was sich schon an den Titeln einiger Studien zur Historizität des Konzeptes zeigt: Postuliert werden die ,Geburt', die ,Entdeckung' oder der ,Aufstieg ${ }^{6}$ des Modus in einem bestimmten Moment der Literaturgeschichte. ${ }^{2}$

Allerdings ist bereits die Frage danach, wann dieser Moment stattgefunden haben soll, stark umstritten und Gegenstand fortwährender und interdisziplinärer Diskussionen. So geht etwa Wolfgang Rösler davon aus, dass Fiktionalität eine

1 Hans Robert Jauß (1983) „Zur historischen Genese der Scheidung von Fiktion und Realität“. Funktionen des Fiktiven. Hgg. Dieter Henrich/Wolfgang Iser. München: Fink. S. 423-431, hier S. 423.

2 So fragen Wolfgang Rösler und Walter Haug jeweils nach der ,Entdeckung' der Fiktionalität, bei Finkelberg 2000 ist von ,Geburt“ („,birth“), bei Gallagher 2006 von ,Aufstieg“ („rise“) die Rede. Vgl. Wolfgang Rösler (1980) „Die Entdeckung der Fiktionalität in der Antike“. Poetica 12. S. 283-319; Walter Haug (2003) Die Wabrheit der Fiktion. Studien zur weltlichen und geistlichen Literatur des Mittelalters und der frühen Neuzeit. Tübingen: Niemeyer. S. 128-144; Margalit Finkelberg (1998) The Birth of Literary Fiction in Ancient Greece. Oxford: Clarendon Press; Catherine Gallagher (2006) „The Rise of Fictionality“. The Novel. Vol 1. Hg. Franco Moretti. Princeton/Oxford: Princeton Univ. Press. S. 336-363. 
Entdeckung der Antike gewesen sei, wohingegen Walter Haug diese Innovation für das 12. Jahrhundert reklamiert. ${ }^{3}$ Dem widerspricht wiederum die wirkungsreiche These, das moderne Fiktionalitätskonzept habe sich erst im 18. Jahrhundert vollständig entfalten können, entweder parallel zur Entwicklung des modernen Autonomiekonzepts ${ }^{4}$ oder in Verbindung mit dem beginnenden Siegeszug des modernen, insbesondere des englischsprachigen Romans. ${ }^{5}$ Catherine Gallagher vermerkt dazu: „[...] the nature of fictionality changed so dramatically in these mideighteen-century British narratives that they do constitute a new form." ${ }^{\text {"6 Die Gründe }}$ für eine solche umfassende Wende in der literarischen Praxis werden dann meistenteils in medialen, kulturellen oder epistemischen Umbrüchen gesucht.

Die Diskussionen beschränken sich allerdings nicht auf die Frage nach der Datierung und den Gründen dieser Wende; vielmehr wird gegen die Vorstellung, Fiktionalität sei als Konzept historisch zu relativieren, auch generell Einspruch erhoben: Es erscheint durchaus zweifelhaft, ob es sich bei Fiktionalität tatsächlich um einen Modus handelt, der zu einem bestimmten Zeitpunkt als kognitive Fähigkeit oder konzeptuelles Wissen, entwickelt' werden musste, ob es also überhaupt einen Moment der Emergenz gegeben hat. Dies würde auch die Frage nach der Ausbildung eines Fiktionsbewusstseins nach sich ziehen: Stand die kognitive Möglichkeit des literarischen Erfindens und einer entsprechenden Rezeptionskompetenz allen historischen Gesellschaften zur Verfügung oder musste sie sich in einem evolutionären Prozess erst etablieren?

In seiner Studie Before Fiction argumentiert Nicholas Paige vehement gegen die Vorstellung, der Mangel an autonom erfundenen Figuren in der vormodernen Literatur sei daraus zu erklären, dass diese Kulturen ,konzeptuell unterbelichtet ${ }^{6}$ („,conceptually short-changed“) waren. ${ }^{7}$ Griechen und Römer etwa seien durchaus in der Lage gewesen, sich vollkommen erfundene Figuren vorzustellen, allerdings habe diese Form der Erfindung eben nicht den Konventionen entsprochen, welche die Angemessenheit literarischer Praktiken reglementierten:

If the Aristotelian critical tradition did not sanction the use of invented heroes, this was not because they didn't have the right ,mental equipment', but - much less dramatically

- because they reasoned that heroes should be taken from history. ${ }^{8}$

\footnotetext{
Vgl. Rösler (1980), Haug (2003) und Jauss (1983).

4 Vgl. Hans-Edwin Friedrich (2009) „Fiktionalität im 18. Jahrhundert. Zur historischen Transformation eines literaturtheoretischen Konzepts“. Grenzen der Literatur. Zum Begriff und Phänomen des Literarischen. Hgg. Simone Winko/Fotis Jannidis/Gerhard Lauer. Berlin/New York: de Gruyter. S. 338-373.

5 Vgl. Ian Watt (1957) The Rise of the Novel. Studies in Defoe, Richardson and Fielding. London: Chatto \& Windus; Lennard J. Davis (1983) Factual Fictions. The Origins of the English Novel. New York: Columbia Univ. Press.

6 Gallagher (2006). S. 336.

7 Nicholas Paige (2011) Before Fiction. The Ancien Régime of the Novel. Philadelphia: University of Philadelphia Press. S. 27.

8 Ebd.
} 
Paige empfiehlt, die Geschichte der Fiktionalität als Geschichte sich verändernder Praktiken zu schreiben und nicht im Sinne dramatischer kognitiver Umbrüche. ${ }^{9}$ Das ändert aber nichts an der Tatsache, dass die Art der Verwendung von literarischer Erfindung historisch stark variiert. Es mag sich um eine Kulturtechnik handeln, die Menschen in allen Zeiten zur Verfügung stand, der allerdings ein je unterschiedlicher Wert beigemessen wurde, was Quantität und Qualität der Praktik beeinflussen musste. In Antike und Mittelalter werden literarische Erfindungen anders zum Einsatz gebracht als in der Moderne, im europäischen Abendland anders als etwa im arabischen Kulturraum. So beruhen zum Beispiel Figuren wie Hans Castorp oder Leopold Bloom, denen kein historisches Substrat zugrunde liegt, auf einer Verwendung von Fiktionalität, die sich erst spät in der Literaturgeschichte durchgesetzt hat (auch wenn sie vorher bereits existierten).

Vor dem Hintergrund solcher Diskussionen beschäftigen sich die gesammelten Beiträge dieses Bandes mit den Fragen und Problemen, die eine historische Untersuchung der Fiktionalität aufwirft. Sie verstehen sich als methodologische und theoretische Vorarbeiten zu einer ,Geschichte der Fiktionalität'. Dass es sich bei dieser Aufgabe um ein Desiderat handelt, wurde in der jüngeren Forschung immer wieder verstärkt angemahnt. So vermerkt etwa schon Frank Zipfel in seiner Studie Fiktion. Fiktivität. Fiktionalität: „In der Forschung gibt es bisher nur einige wenige Ansätze einer historischen Betrachtung von literarischer Fiktion. " ${ }^{10}$ Und auch Hans-Edwin Friedrich weist in seiner Untersuchung zum Fiktionalitätskonzept des 18. Jahrhunderts darauf hin, dass „eine Geschichte der Fiktionalität“ sich "anhand der vorliegenden Forschung erst in vagen Umrissen" abzeichne. ${ }^{11}$

Ein Hindernis, das einer historischen Synthese der Entwicklung von Fiktionskonventionen bislang häufig im Wege stand und das es zu überwinden gilt, ist sicherlich der umfassende interdisziplinäre Anspruch, den ein solches Projekt mit sich bringt. Bisherige Beiträge zum Thema sind notgedrungen auf die philologische und historische Ausrichtung des jeweiligen Forschungsfeldes beschränkt. Eine vollständige Geschichte der Fiktionalität wäre allerdings auf eine Perspektive angewiesen, die Expertise in allen historischen und philologischen Disziplinen miteinschließt. Dazu müssten selbstverständlich auch Fiktionalitätspraktiken jenseits der in der Forschung kanonisierten, westlichen' Kulturen betrachtet werden. Der vorliegende Band vertritt nicht den Anspruch, diese Anforderungen umfänglich einzulösen, sondern möchte vielmehr Ansätze bieten, die zu einem weiteren Nachdenken über die Geschichtlichkeit des Fiktionalitätskonzepts anregen. Die Beiträge beschäftigen sich mit Teilaspekten und Problemfeldern der Fiktionsgeschichte von der Antike bis in die Gegenwart. Terminologische und theoretische

9 Ebd. S. 26: „This is not to say, however, that human cognition makes a leap, only that people's literary behavior changes - generally speaking."

10 Frank Zipfel (2001) Fiktion, Fiktivität, Fiktionalität. Analysen zur Fiktion in der Literatur und zum Fiktionsbegriff in der Literaturwissenschaft. Berlin: Erich Schmidt. S. 285.

11 Friedrich (2009). S. 338. 
Fragen werden dabei genauso in den Blick genommen wie methodologische Aufgaben und Probleme (etwa in Bezug auf die Rekonstruktion kultureller Grundlagen oder die mitunter prekäre Quellenlage). Dabei haben sich die folgenden übergreifenden Fragen und Aufgaben ergeben:

1. Eine grundsätzliche Herausforderung, der sich die historische Fiktionsforschung stellen muss, ist der Entwurf einer tragfähigen Definition des Gegenstandes. Die Debatte darüber, was ,Fiktionalität' eigentlich bedeutet, ist nach wie vor nicht abgeschlossen. Eine diachrone Perspektive erscheint zunächst dazu geeignet, diese Debatte zu verkomplizieren; so muss die anachronistische Applikation eines modernen Begriffs für literarischer Erfindungen in allen Zeiten vermieden werden. ${ }^{12}$ Gleichzeitig birgt etwa der Versuch, das Phänomen grundsätzlich aus den zeitgenössischen Diskussionen und Begriffen zu entwickeln, die Gefahr eines historischen Relativismus und einer daraus folgenden terminologischen Beliebigkeit, die eine zumindest ansatzweise kohärente diachrone Erzählung unmöglich macht. Gerade die historische Perspektive ist auf eine stabile Definition in besonderer Weise angewiesen. Vor diesem Hintergrund erscheint die, institutionel$1 e^{6}$ Theorie der Fiktionalität, die sich inzwischen in vielen fiktionstheoretischen Arbeiten durchgesetzt hat, als angemessene Arbeitsgrundlage. Demnach bezeichnet Fiktionalität eine soziale Institution, eine „Praxis des geteilten Konventionsoder Regelwissens". ${ }^{13}$ Diesem Modell liegt ein kommunikationstheoretisch fundierter Ansatz zugrunde, der davon ausgeht, dass ein fiktionaler Geltungsanspruch über entsprechende Signale vermittelt wird. ${ }^{14}$ Dieser Geltungsanspruch verspricht die Fiktivität einiger Elemente innerhalb der Erzählung und reklamiert damit gewisse epistemologische, ästhetische und ethische Lizenzen in der Darstellung. ${ }^{15}$ Gleichzeitig wird eine fiktionsangemessene Rezeptionshaltung eingefordert. Der Vorteil einer institutionellen Theorie von Fiktionalität für eine historische Perspektive liegt darin, dass die Konventionen, welche die Struktur der Kommunikationssituation bestimmen als kontextabhängig bestimmt werden. Wie ein Geltungsanspruch begründet und signalisiert wird, ist von den Normen

12 So weist etwa Jan-Dirk Müller darauf hin, dass in Studien zur Fiktionalität im Mittelalter, ein „an der Moderne abgelesener Begriff von Fiktionalität“ auf unangemessene Art und Weise verwendet wird, vgl. Ders. (2004) „Literarische und andere Spiele. Zum Fiktionalitätsproblem in vormoderner Literatur“. Poetica 36. S. 281-312, hier S. 283.

13 Tobias Klauk/Tilmann Köppe (2014) „Bausteine einer Theorie der Fiktionalität“. Fiktionalität. Ein interdisziplinäres Handbuch. Hgg. Dies. Berlin/Boston: de Gruyter. S. 3-31, hier S. 7, vgl. auch Tilmann Köppe (2014) „Die Institution der Fiktionalität“, im selben Band S. 35-49. Köppe führt die institutionelle Theorie insbesondere auf Peter Lamarque/ Stein Haugom Olsen (1994) Truth, Fiction, and Literature. A Philosophical Perspective. Oxford: Clarendon Press zurück.

14 Vgl. Frank Zipfel (2014) „Fiktionssignale“. Fiktionalität. Ein interdisziplinäres Handbuch. Hgg. Tobias Klauk/Tilmann Köppe Berlin/Boston: de Gruyter. S. 97-124.

15 Vgl. Johannes Franzen (2017) „Ein Recht auf Rücksichtslosigkeit. Die moralischen Lizenzen der Fiktionalität“. Non-Fiktion 12. S. 31-48. 
abhängig, nach denen sich die Kommunikationsteilnehmer richten. Ob bzw. in welchem Umfang eine Gesellschaft bereit ist, den Status der Fiktionalität und die damit verbundenen Lizenzen zuzulassen, lässt sich durch eine Rekonstruktion der entsprechenden kommunikativen Kontexte bestimmen. ${ }^{16}$

2. Ein genuin methodisches Problem ergibt sich für die Rekonstruktion der historischen Kommunikationssituationen: Wie kann aus der Retrospektive festgestellt werden, welchen Geltungsanspruch ein Text vertrat und ob er als fiktional oder faktual gelesen wurde? Quellenprobleme ergeben sich dort, wo es an einschlägigen Rezeptionszeugnissen fehlt bzw. wo sich die Deutung konventionalisierter Fiktionssignale als problematisch erweist. Zudem muss danach gefragt werden, ob historische Literaturtheorie und tatsächliche literarische Praxis miteinander konvergieren, ob also etwa die Existenz einer poetologischen Diskussion über Funktion und Legitimität von Fiktionalität - wie sie etwa im Roman des späten 17. Jahrhunderts geführt wird ${ }^{17}$ - als Indikator eines verbreiteten Fiktionsbewusstseins gelten kann. So hat Tilmann Köppe darauf hingewiesen, dass gerade die Abwesenheit einer expliziten Debatte über die Angemessenheit einer literarischen Praxis einen Indikator für die ,Selbstverständlichkeit ${ }^{6}$ dieser Praxis darstellen kann: „Man muss daher damit rechnen, dass das explizit Gesagte gerade nicht dem konventionellen Regelfall entspricht bzw. diesen gerade nicht repräsentiert."18 Eine historische Fiktionsforschung muss sich der Aufgabe stellen, repräsentative Quellen zu finden, die der Alterität von Produktions- und Rezeptionskontext gerecht werden. Nur so lassen sich die unterschiedlichen Formen von Geltungsansprüchen und Rezeptionshaltungen angemessen bestimmen. Damit geht die Frage nach dem historisch und kulturell variablen Status der Fiktionskompetenz einher. ${ }^{19}$ Wie geläufig war den Rezipienten die Institution der Fiktionalität? Gab es innerhalb dieser Gruppe Unterschiede in der Akzeptanz und im Besitz des entsprechenden Regelwissens? Vor dem Hintergrund dieser Fragen kommt etwa Mark Chinca zu der Feststellung:

Der Mediävist aber, der die Fiktionalität des höfischen Romans im frühen 13. Jahrhundert pragmatisch erklären möchte, stößt auf das Problem, daß Fiktionalität zu dieser Zeit

16 So bereits Tilmann Köppe (2014) „Fiktionalität in der Neuzeit“. Fiktionalität. Ein interdisziplinäres Handbuch. Hgg. Tobias Klauk/Ders. Berlin/Boston: de Gruyter. S. 419-440, hier S. 420: „Die institutionelle Theorie der Fiktionalität stellt einen flexiblen Rahmen zur Beschreibung historischer Wandlungen von Institutionen zur Verfügung. Das Wissen um Regeln und Konventionen - und damit das Regel bzw. Konventionsset selbst - wird tradiert und kann sich im Laufe der Zeit in vielfältiger Weise ändern."

17 Vgl. Christian Berthold (1993) Fiktion und Vieldeutigkeit. Zur Entstehung moderner Kulturtechniken des Lesens im 18. Jabrhundert. Tübingen: Niemeyer. S. 23-32 und Stefan Trappen (1998) „Fiktionsvorstellungen der Frühen Neuzeit. Über den Gegensatz zwischen ,fabula‘ und ,historia' und seine Bedeutung für die Poetik“. Simpliciana 20. S. 137-143.

18 Köppe (2014) „Neuzeit“. S. 421.

19 Norbert Groeben/Carsten Dutt (2011) „Fiktionskompetenz“. Handbuch Erzäblliteratur. Theorie, Analyse, Geschichte. Hg. Matías Martinez. Weimar: Metzler. S. 63-67. 
noch nicht selbstverständlich war, die mit ihr verbundenen Haltungen und Verpflichtungen noch nicht völlig eingeübt waren. ${ }^{20}$

Auch für Chinca verweisen die häufigen poetologischen Passagen in den Romanen über die pragmatischen Möglichkeiten der Fiktionalität also auf den Sonderstatus einer noch nicht etablierten literarischen Technik, der hier in direktem Verweis auf die historische Fiktionskompetenz eingeschätzt wird.

3. Das jeweilige Wirklichkeitsmodell einer historischen Gesellschaft muss rekonstruiert werden: Die Geltungsansprüche ,Fiktionalität ${ }^{`}$ und ,Faktualität ${ }^{\star}$ bezeichnen zunächst Versprechen von Referenz bzw. Nichtreferenz und beziehen sich demnach auf eine historisch und kulturell bedingte Alltagswirklichkeit. Dieses Wirklichkeitskonzept definiert Zipfel in Anlehnung an Nelson Goodman als das „was den Mitgliedern einer Gesellschaft als wirklich oder real gilt." ${ }^{21}$ Wird dieser soziale Konstruktivismus anerkannt, so erübrigen sich komplexe und fruchtlose „erkenntnistheoretische Betrachtungen zum Wirklichkeitsbegriff“. Stattdessen wird der Blick frei für eine Historisierung unterschiedlicher Alltagswirklichkeiten. Auch darauf weist Zipfel hin, wenn er vermerkt, dass was „als Wirklichkeit im Sinne von Alltagswelt gilt“, letztlich „immer vom jeweiligen sozial-historischen Kontext“ abhängt: „Insofern ist natürlich auch das, was konkret als Fiktion gilt - insofern es an das Konzept der Wirklichkeit gekoppelt ist -, historisch variabel."22 Für die Geschichte der Fiktionalität ergeben sich daraus Fragen wie: Welche Elemente eines Textes gehören zur Weltwahrnehmung der Rezipienten und welche Elemente werden als fantastisch/nicht-existent wahrgenommen? Diese Fragen richten sich auf die Alterität historischer Weltbilder: Nur weil bestimmte Elemente älterer Texte, wie etwa Drachen und andere Fabelwesen, heute als nicht-existent angesehen werden, heißt das nicht, dass diese Texte fiktional waren. Auch in diesem Zusammenhang zeigt sich die Tragfähigkeit institutioneller Modelle, wie etwa Sonja Glauch in ihrer Studie zur Fiktionalität im Mittelalter anmerkt:

Gerade ,alte ${ }^{6}$ Texte zeigen: es kann nicht darum gehen, wie ein heutiger Leser oder Interpret ihren Wahrheitsgehalt oder Wahrheitsanspruch beurteilt. Der intendierte zeitgenössische Leser oder Zuhörer und sein intendierter Umgang mit dem Text muss das Kriterium sein. $^{23}$

4. Eine Geschichte der Fiktionalität bedarf zudem einer Integration in die Literatur- und Mediengeschichte. Es muss etwa geklärt werden, welche gattungsgeschichtlichen Entwicklungen oder poetologischen Revolutionen die Verbreitung des Konzepts beeinflusst haben. So schreiben Lenard Davis in Factual Fictions, Paige in Before Fiction und Gallagher in "The Rise of Fictionality" Fiktionsgeschichte als Romangeschichte. Dem von Ian Watts festgestellten ,Rise of the no-

20 Mark Chinca (2003) „Mögliche Welten. Alternatives Erzählen und Fiktionalität im Tristanroman Gottfrieds von Straßburg“. Poetica 35. S. 307-333, hier S. 313.

21 Zipfel (2001). S. 75.

22 Ebd. S. 76.

23 Sonja Glauch (2014) „Fiktionalität im Mittelalter; Revisited“. Poetica 46. S. 85-139, hier S. 91. 
vel' scheint aus dieser Perspektive große historische Bedeutung für die Entwicklung eines modernen Fiktionsverständnisses zuzukommen. In diesem Zusammenhang muss allerdings vor der Gefahr einer gewissen Gattungsblindheit der Fiktionsforschung gewarnt werden: Die Frage nach dem Status literarischer Erfindung stellt sich unter anderem auch in Bezug auf die Geschichte des Dramas oder der Lyrik. Zumindest scheint es aber Leitgattungen zu geben, die jeweils für einen bestimmten Zeitraum die Verwendung von Fiktionalität sowie die Debatte darüber bestimmten. Eine weitere literaturgeschichtliche Entwicklung, die als Faktor fiktionshistorischer Veränderungen vorgeschlagen wurde, ist der Aufstieg des modernen Autonomieparadigmas. In diesen Kontext verortet Hans-Edwin Friedrich die „Transformation des Fiktionskonzepts im 18. Jahrhundert“. Dabei handele es sich „weder um die Entdeckung oder Erfindung des Fiktionsbewusstseins noch um ein endlich erreichtes Telos, sondern um eine Veränderung, die von der Autonomisierung als Folge der funktionalen Ausdifferenzierung der Kunst bewirkt wird." 24 Über solche Erklärungsansätze hinaus kann gefragt werden, inwiefern sich Fiktionsgeschichte als Geschichte literarischer Stoffe schreiben lässt. Neben solchen gattungs- und poetologiegeschichtlichen Einordnungen, muss in die historische Untersuchung der Fiktionalität auch der mediale Wandel miteinbezogen werden: Wie beeinflussten entsprechende Innovationen - Entwicklung von Schriftlichkeit, bildliche Repräsentationstechniken, Fotographie, Film etc. - die Geschichte der Fiktionalität?25

5. Inwiefern lässt sich darüber hinaus die Geschichte der Fiktionalität als Geschichte ihrer Bewertung schreiben? Diese Frage betrifft die ,Legitimität der Fiktion'. ${ }^{6}$ Ein geläufiges Narrativ erzählt etwa die europäische/abendländische Wertungsgeschichte der Fiktionalität als eine Durchsetzungsgeschichte: von der Dichterverbannung Platons, über das christliche Fiktionsmisstrauen des Mittelalters und die Romanschelte des 17. Jahrhunderts bis hin zum Aufstieg der Fiktionalität als dem eigentlichen Literarizitätsgaranten der Moderne. ${ }^{27}$ Auch hier ergeben sich offensichtliche Fragen nach Konjunkturen und Brüchen. So zeigen Debatten um stoffgeschichtliche Fiktionsverbote - wie die zeitgenössische Skepsis gegenüber erfundenen Shoa-Narrativen -, dass Fiktionskritik keineswegs ein genuin vormodernes Phänomen darstellt. ${ }^{28}$ Stattdessen wird deutlich, dass die ethische

24 Friedrich (2009). S. 339.

$25 \mathrm{Zu}$ den Problemen einer ,transmedialen Fiktionstheorie ${ }^{6}$ vgl. Jan-Noël Thon (2014) „Fiktionalität in Film- und Medienwissenschaft“. Fiktionalität. Ein interdisziplinäres Handbuch. Hgg. Tobias Klauk/Ders. Berlin/Boston: de Gruyter. S. 434-466.

26 So der Titel einer Studie von Aleida Assmann (1980) Die Legitimität der Fiktion. Ein Beitrag zur Geschichte der literarischen Kommunikation. München: Fink.

27 Vgl. Gerhard Sauder (1976) „Argumente der Fiktionskritik 1680-1730 und 1960-1970“. Germanisch-Romanische Monatsschrift 26. S. 129-140

$28 \mathrm{Zu}$ den ethischen Problemen von Holocaust-Literatur und Autorschaft vgl. Alvin $\mathrm{H}$. Rosenfeld (2004) „The Problematics of Holocaust Literature“. Literature of the Holocaust. Hg. Harold Bloom. Philadelphia: Chelsea House. S. 21-48. 
und ästhetische Angemessenheit der Fiktionalität in allen Zeiten umstritten war. Insgesamt erweisen sich die Statusbeschreibungen ,Fiktionalität ${ }^{6}$ und ,Faktualität als traditionsreiches Konkurrenzverhältnis mit einer eindeutigen evaluativen Komponente, die historischen Konjunkturen unterworfen ist. ${ }^{29}$

6. Eine diachrone Betrachtung umfasst auch die Frage nach der historischen Karriere von Texten: Die Literaturgeschichte kennt eine Vielzahl von Fällen, in denen Texte, die ursprünglich mit dem Geltungsanspruch der Faktualität geschrieben wurden, von Rezipienten späterer Zeiten als fiktional gelesen werden. Dies bezeichnet nicht selten einen Rettungsversuch historischer Texte, welche dem Wirklichkeitsmodell der eigenen Zeit nicht mehr gerecht werden können, und denen durch die Umdeutung zu fiktionalen Werken ein neuer Wert zugeschrieben wird. Das gilt etwa für die antike Homer-Rezeption, die den Vorwurf der vorwissenschaftlichen Fehlerhaftigkeit der göttlich inspirierten Dichterwerke umzukehren versucht. So vermerkt Heinz Schlaffer zum Status der antiken Poesie: „Den Gegnern gilt das Fiktive an ihr als sicheres Indiz des Falschen; die Apologeten müssen daher die Fiktion zur harmlosen Verkleidung des Wahren, Guten und Ewigen umdeuten. “30 Ähnlich verhält es sich mit der Mythenrezeption des christlichen Mittelalters, dem der Status der Fiktionalität dazu diente, den Traditionsverbrauch zu begründen. Dazu wiederum Schlaffer: „Im Mittelalter galt Vergil geradezu als christlicher Dichter und Ovid immerhin als ein moralischer." 31 Solche Phänomene werfen im Wesentlichen die Frage nach dem möglichen historischen Wandel des Fiktionalitäts- oder Faktualitätsstatus eines Textes auf. Dieser Wandel muss nicht auf den Wechsel von faktual zu fiktional festgelegt werden: So können etwa im Fall von Schlüsselromanen die primären faktualisierenden Lektüren mit dem Verlust des Hintergrundwissens verschwinden.

$*$

Mit Fragen wie diesen beschäftigen sich die Beiträge dieses Bandes, die keineswegs Anspruch auf Vollständigkeit erheben. Es wurde allerdings versucht, die verschiedenen Probleme einer Historisierung von Fiktionalität aus einer größtmöglichen Vielfalt disziplinärer und geschichtlicher Perspektiven zu beleuchten. Eröffnet wird der Band durch die theoretischen Überlegungen Benjamin GITTELs, der in den Diskussionen zur Geschichte der Fiktionalität zwei zentrale Genres ausmacht: fiktionale, konstitutiv wirklichkeitsbezogene Genres einerseits und skalar fiktionale Genres andererseits. Gefragt wird, ob und inwiefern diese Genrety-

29 Vgl. Johannes Franzen (2016) „Mehr Bild als Roman‘. Fiktionalität, Faktualität und das Problem der Bewertung“. Der Deutschunterricht 4. S. 20-28.

30 Heinz Schlaffer (1990) Poesie und Wissen. Die Entstehung des ästhetischen Bewußtseins und der philologischen Erkenntnis. Frankfurt am Main: Suhrkamp. S. 82.

31 Ebd. S. 85. 
pen eine Herausforderung für systematisch ausgerichtete Fiktionalitätstheorien darstellen. So können nicht nur Ziele und Adäquatheitsbedingungen unterschiedlicher Fiktionalitätstheorien beleuchtet, sondern auch mögliche Verhältnisse fiktions- und genrespezifischer Praktiken diskutiert werden.

Wolfgang RÖSLER zeichnet den Wandel in der Auffassung von der Rolle des Dichters im antiken Griechenland nach und fragt hierbei nach dem spezifischen Beitrag der dramatischen Dichtung für die Profilierung eines Fiktionalitätskonzepts. Zu dessen Institutionalisierung bedarf es der Modifizierung und Aufgabe traditioneller Umgangspraktiken und einer grundsätzlich veränderten Einstellung zur Dichtung als Fiktion. Dabei lassen sich bereits in antiken Konzeptionen Analogien zu modernen Theorien erkennen.

Henrike MANUWALD geht am Beispiel des Drachens in der volkssprachigen Literatur des Mittelalters der Frage nach, inwieweit die Plausibilität des Erzählten in Relation zur historischen Wirklichkeitserfahrung steht. Im Umgang mit dem Wunderbaren und Fremden lassen sich für verschiedene Gattungen unterschiedliche Fiktionalitätskonzepte sowie konkurrierende Wahrheitsansprüche konstatieren. In Auseinandersetzung mit aktuellen Positionen der mediävistischen Debatte plädiert sie folglich für eine grundsätzliche Historisierung der Kategorien faktual/fiktional.

Ebenfalls mit Problemen der Fiktionalität in mittelalterlicher Literatur beschäftigen sich Sebastian KLEINSCHMIDT und Verena SPOHN, die sich dem in fiktionstheoretischen Studien bislang unterrepräsentierten Bereich religiöser Literatur nähern. Am Beispiel mittelalterlicher Visionsnarrative zeigen sie, wie sich das eigentliche Visionsgeschehen durch die Setzung eines narrativen Rahmens als eigenständiger Textraum etabliert, was den mittelalterlichen Autoren kreative Spielflächen zur Erprobung des Fiktionalen eröffnet, ohne dem religionsdidaktischen Anspruch der Gattung zu schaden.

Den für eine umfassende Geschichte der Fiktionalität bedeutsamen Blick auf nicht-europäische Kulturen richtet Isabel TORAL-NIEHOFF in ihrem Beitrag zur arabischen Literatur zwischen dem 8. und 10. Jahrhundert. Sie widmet sich dabei dem Phänomen hybrider Textformen. Obwohl das arabische Mittelalter Konzepte von Fiktionalität grundsätzlich ablehnte, lässt sich an Texten der kompilatorisch angelegten $a d a b$-Literatur eine funktionale Verwendung von Fiktionalisierungsstrategien erkennen, die auch Parallelen zur europäischen mittelalterlichen Literatur aufweist.

Roman KUHN widmet sich den paratextuellen Faktualitätssignalen in der englischen Romanliteratur des 18. Jahrhunderts. Er arbeitet heraus, dass gerade solche Paratexte, die auf die Wahrheit der Erzählung insistieren, als konventionelle Muster selbst wieder zu Fiktionsalitätssignalen werden können. Die zur Codierung dieser spielerischen Bewegungen zentrale Fähigkeit hermeneutischer Paratextlektüre wird exemplarisch an Daniel Defoes The Life and Strange Surprizing Adventures of Robinson Crusoe vorgeführt. 
Der Beitrag von Christopher MEID analysiert anhand romantheoretischer Stellungnahmen von Christian Friedrich Hunold bis Christoph Martin Wieland den allmählichen Wandel des Fiktionalitätsverständnisses im 18. Jahrhundert. Im Zentrum des Interesses steht dabei das Verhältnis von Roman und Historie: Während sich die Texte der ersten Jahrhunderthälfte über den Bezug zu verbürgten Fakten legitimieren, spielt Wieland in der Geschichte des Agathon mit dieser Tradition und ironisiert sie.

Frank ZIPFEL liest in seinem Beitrag Madame de Staëls Essai sur les fictions als fiktionstheoretische Abhandlung, die trotz völlig anderer Terminologie zahlreiche Ähnlichkeiten zur institutionellen Theorie der literarischen Fiktion aufweist. Dabei zeigt er Verbindungen zentraler fiktionstheoretischer Begriffe wie dem der ,Imagination' zu modernen Theorien ebenso auf wie signifikante Unterschiede zur heutigen Diskussion. Zipfel würdigt im Vergleich mit einigen zeitgenössischen Poetologien und Ästhetiken den Essai als exemplarischen Ausdruck der Fiktionstheorien des 18. Jahrhunderts.

In seiner Auseinandersetzung mit Wilhelm Diltheys literaturtheoretischen Arbeiten rekonstruiert Mathis LESSAU dessen produktionsbezogene Fiktionalitätstheorie. Durch eine komplexe Transformation realer Erfahrungsinhalte könne die dichterische Phantasie eine fiktive Welt schaffen, die zwar nur einen Schein von Wirklichkeit erzeugt, aber dennoch von kognitivem Wert für das Verständnis des Lebens sei. Der exegetische Bezug von Fiktionen zum wirklichen Leben zeige sich für Dilthey unter anderem in den widersprüchlich anmutenden emotionalen Reaktionen von Lesern auf Dichtungen. Vor dem Hintergrund aktuellerer Ansätze zum sogenannten ,Paradoxon der Fiktion' wird Diltheys Verständnis der emotionalen Kraft von fiktionaler Literatur analysiert und literaturphilosophisch eingeordnet.

Marc WURICH zeigt am Beispiel von naturalistischem Roman und sozialer Reportage, wie um 1900 neue bzw. modifizierte Textgenres mit ausgeprägtem Wirklichkeitsbezug konventionalisierte Rezeptionspraktiken herausforderten. Die exklusive Zugänglichkeit der hier dargestellten Milieus eröffnete den Autoren spezifische Möglichkeiten zur Funktionalisierung. Während die textkonstitutive Heteroreferentialität den literarischen Fiktionen auch einen dokumentarischen Anspruch verleiht und die Teilnahme an faktualen Diskursen legitimieren soll, bedienen sich die journalistischen Texte mit wirkungsästhetischer Absicht auch fiktionalisierender Verfahren.

In ihrem Beitrag zu kontrafaktischen Narrativen geht Françoise LAVOCAT insbesondere anhand von Beispielen aus der Literatur seit 1990 der Frage nach, worin sich alternative Geschichtsentwürfe und Romanfiktionen unterscheiden lassen. Da dem Kontrafaktischen in beiden Textformen ein fiktionaler Status zugeordnet ist, geraten hierbei vornehmlich epistemische Aspekte in den Fokus der Untersuchung. Neben differierenden Erkenntniszielen lassen sich für die jeweils dargestellten möglichen Welten auch voneinander abweichende axiologische Modalitäten feststellen. 
Abschließend geht Johannes FRANZEN versuchsweise auf das traditionsreiche Konzept der Fiktionskritik ein. Hierbei steht vor allem die Frage im Mittelpunkt, inwiefern mit der Vorstellung einer überlegenen ,Wahrheit der Fiktion' eine analoge Furcht vor einer ,Unwahrheit der Fiktion' einhergeht. Einige Aspekte dieser Skepsis gegenüber der Freiheit des literarischen Erfindens werden anhand von historisch und kulturell weit auseinanderliegenden Beispielen untersucht, um so die zugrundeliegende Argumentationsstruktur der Fiktionskritik herauszuarbeiten. Aus einer historischen Perspektive wird gezeigt, dass entsprechende Einschränkungen auch in der Gegenwart existieren.

$*$

Die in diesem Band gesammelten Beiträge beruhen auf einer Konferenz, die vom 7. bis zum 8. November 2014 am Freiburg Institute for Advanced Studies (FRIAS) stattfand. Die Konferenz wurde vom Graduiertenkolleg 1767 (,Faktuales und Fiktionales Erzählen') ausgerichtet und finanziert. Stellvertretend für das Graduiertenkolleg gilt unser herzlicher Dank der Sprecherin Prof. Dr. Monika Fludernik, insbesondere auch für die Aufnahme der Publikation in die Buchreihe des GRK.

\section{Literatur}

Assmann, Aleida (1980) Die Legitimität der Fiktion. Ein Beitrag zur Geschichte der literarischen Kommunikation, München: Fink.

Berthold, Christian (1993) Fiktion und Vieldeutigkeit. Zur Entstehung moderner Kulturtechniken des Lesens im 18. Jahrbundert. Tübingen: Niemeyer. S. 23-32.

Chinca, Mark (2003) „Mögliche Welten. Alternatives Erzählen und Fiktionalität im Tristanroman Gottfrieds von Straßburg". Poetica 35. S. 307-333.

Davis, Lennard J. (1983) Factual Fictions. The Origins of the English Novel. New York: Columbia Univ. Press.

Finkelberg, Margalit (1998) The Birth of Literary Fiction in Ancient Greece. Oxford: Clarendon Press.

Franzen, Johannes (2017) „Ein Recht auf Rücksichtslosigkeit. Die moralischen Lizenzen der Fiktionalität“. Non-Fiktion 12. S. 31-48.

--- (2016) „Mehr Bild als Roman`. Fiktionalität, Faktualität und das Problem der Bewertung“. Der Deutschunterricht 4. S. 20-28.

Friedrich, Hans-Edwin (2009) „Fiktionalität im 18. Jahrhundert. Zur historischen Transformation eines literaturtheoretischen Konzepts“. Grenzen der Literatur. Zum Begriff und Pbänomen des Literarischen. Hgg. Simone Winko/Fotis Jannidis/Gerhard Lauer. Berlin/New York: de Gruyter. S. 338-373.

Gallagher, Catherine (2006) „The Rise of Fictionality“. The Novel. Vol 1. Hg. Franco Moretti. Princeton/Oxford: Princeton Univ. Press. S. 336-363.

Glauch, Sonja (2014) „Fiktionalität im Mittelalter; Revisited“. Poetica 46. S. 85-139. 
Groeben, Norbert/Carsten Dutt (2011) „Fiktionskompetenz“. Handbuch Erzäblliteratur. Theorie, Analyse, Geschichte. Hg. Matías Martinez. Weimar: Metzler. S. 63-67.

Haug, Walter (2003) Die Wabrheit der Fiktion. Studien zur weltlichen und geistlichen Literatur des Mittelalters und der frühen Neuzeit. Tübingen: Niemeyer.

Jauß, Hans Robert (1983) „Zur historischen Genese der Scheidung von Fiktion und Realität“. Funktionen des Fiktiven. Hgg. Dieter Henrich/Wolfgang Iser. München: Fink. S. 423-431.

Klauk, Tobias/Tilmann Köppe (2014) „Bausteine einer Theorie der Fiktionalität“. Fiktionalität. Ein interdisziplinäres Handbuch. Hgg. Dies. Berlin/Boston: de Gruyter. S. 3-31.

Köppe, Tilmann (2014) „Fiktionalität in der Neuzeit“. Fiktionalität. Ein interdisziplinäres Handbuch. Hgg. Tobias Klauk/Ders. Berlin/Boston: de Gruyter. S. 419-440.

--- (2014) „Die Institution der Fiktionalität“. Fiktionalität. Ein interdisziplinäres Handbuch. Hgg. Tobias Klauk/Ders. Berlin/Boston: de Gruyter S. 35-49.

Lamarque, Peter/Stein Haugom Olsen (1994) Truth, Fiction, and Literature. A Philosophical Perspective. Oxford: Clarendon Press.

Müller, Jan-Dirk (2004) „Literarische und andere Spiele. Zum Fiktionalitätsproblem in vormoderner Literatur". Poetica 36. S. 281-312.

Paige, Nicholas (2011) Before Fiction. The Ancien Régime of the Novel. Philadelphia: University of Philadelphia Press.

Rosenfeld, Alvin H. (2004) „The Problematics of Holocaust Literature“. Literature of the Holocaust. Hg. Harold Bloom. Philadelphia: Chelsea House.

Rösler, Wolfgang (1980) „Die Entdeckung der Fiktionalität in der Antike“. Poetica 12. S. 283-319.

Schlaffer, Heinz (1990) Poesie und Wissen. Die Entstehung des ästhetischen Beroußtseins und der philologischen Erkenntnis. Frankfurt am Main: Suhrkamp.

Sauder, Gerhard (1976) „Argumente der Fiktionskritik 1680-1730 und 19601970“. Germansich-Romanische Monatsschrift 26. S. 129-140.

Thon, Jan-Noël (2014) „Fiktionalität in Film- und Medienwissenschaft“. Fiktionalität. Ein interdisziplinäres Handbuch. Hgg. Tobias Klauk/Ders. Berlin/ Boston: de Gruyter. S. 434-466.

Trappen, Stefan (1998) „Fiktionsvorstellungen der Frühen Neuzeit. Über den Gegensatz zwischen ,fabula' und ,historia ${ }^{6}$ und seine Bedeutung für die Poetik“. Simpliciana 20. S. 137-143.

Watt, Ian (1957) The Rise of the Novel. Studies in Defoe, Richardson and Fielding. London: Chatto \& Windus.

Zipfel, Frank (2001) Fiktion, Fiktivität, Fiktionalität. Analysen zur Fiktion in der Literatur und zum Fiktionsbegriff in der Literaturwissenschaft. Berlin: Erich Schmidt.

- (2014) „Fiktionssignale“. Fiktionalität. Ein interdisziplinäres Handbuch. Hgg. Tobias Klauk/Tilmann Köppe. Berlin/Boston: de Gruyter. S. 97-124. 